\title{
Symmetries and Singularities of the Szekeres System
}

\author{
Andronikos Paliathanasis ${ }^{1,2, *}$ and P.G.L. Leach ${ }^{3,4,0}$ \\ ${ }^{1}$ Instituto de Ciencias Físicas y Matemáticas, Universidad Austral de Chile, Valdivia, Chile \\ ${ }^{2}$ Institute of Systems Science, Durban University of Technology, POB 1334 Durban 4000, South Africa. \\ ${ }^{3}$ Department of Mathematics and Institute of Systems Science, \\ Research and Postgraduate Support, Durban University of Technology, \\ POB 1334 Durban 4000, Republic of South Africa \\ ${ }^{4}$ School of Mathematics, Statistics and Computer Science, University of KwaZulu-Natal, \\ Private Bag X54001, Durban 4000, Republic of South Africa
}

\begin{abstract}
The Szekeres system is studied with two methods for the determination of conservation laws. Specifically we apply the theory of group invariant transformations and the method of singularity analysis. We show that the Szekeres system admits a Lagrangian and the conservation laws that we find can be derived by the application of Noether's theorem. The stability for the special solutions of the Szekeres system is studied and it is related with the with the Left or Right Painlevé Series which describes the expansions.
\end{abstract}

PACS numbers:

Keywords: Szekeres system; Silent universe; Lie symmetries; Singularity analysis

Gravitational models with vanishing magnetic part of Weyl curvature tensor and irrotational dust fluid component have been studied by Bruni, Matarrese and Pantano in [1]. Because there is no information dissemination with gravitational or sound waves between the worldlines of neighboring fluid elements these models are called silent universe [2]. The field equations form a system of six first-order ordinary differential equations. The exact solutions at the critical points were found to be Tolman-Bondi, Kantowski-Sachs or Szekeres geometries which can be seen as perturbations of Friedmann-Lemaitre-Robertson-Walker spacetimes [1]. The stability of these solutions was studied in [3] where it was found that solutions which describe pancakelike collapse are stable while those that describe spindlelike collapse are unstable, while in [4] was shown that the spacetimes that follows from the Szekeres system are "partially" locally rotational spacetimes (PLRS). The integrability conditions for the silent universes where studied in [2] wherein a conjecture was given such that "there are no spatially inhomogeneous irrotational dust silent models, whose Weyl curvature tensor is of algebraic Petrov type I".

The field equations of the silent universe comprise a system of six first-order ordinary differential equations ${ }^{1}$ in which the dependent variables are: the energy density of the dust fluid, $\rho$, the expansion rate of the observer, $\theta$, the shear components of the observer, $\sigma_{1}$ and $\sigma_{2}$, and the two components of the electric part of the Weyl curvature tensor, i.e. $E_{1}$ and $E_{2}$. A special case is when the electric parts of the Weyl tensor are equal, i.e., $E_{1}=E_{2}$ as also the shear $\sigma_{1}=\sigma_{2}$. In that case any irrotational dust models with vanishing magnetic field is described by the so-called Szekeres system [5, [6]. The dynamics of the Szekeres system can be found in [7], while a covariant formulation based on the $1+1+2$ decomposition was derived recently in [8].

The equations which form the Szekeres system are

$$
\begin{aligned}
\dot{\rho}+\theta \rho & =0, \\
\dot{\theta}+\frac{\theta^{2}}{3}+6 \sigma^{2}+\frac{1}{2} \rho & =0, \\
\dot{\sigma}-\sigma^{2}+\frac{2}{3} \theta \sigma+\mathcal{E} & =0, \\
\dot{\mathcal{E}}+3 \mathcal{E} \sigma+\theta \mathcal{E}+\frac{1}{2} \rho \sigma & =0,
\end{aligned}
$$

plus the algebraic equation

$$
\frac{\theta^{2}}{3}-3 \sigma^{2}+\frac{(3) R}{2}=\rho
$$

\footnotetext{
*Electronic address: anpaliat@phys.uoa.gr

${ }^{\dagger}$ Electronic address: leach.peter@ucy.ac.cy

1 The system is an algebraic-differential system in which the algebraic equation is related with the curvature of the three-dimensional space.
} 
where a dot means contraction of the covariant derivative $\nabla_{\mu}$, with respect to the timelike four-vector field $u^{\mu}$, such that $\dot{A}=A_{; \mu} u^{\mu}$. However except from the above system the $1+3$ analysis of the field equations provides the spacelike constraints [9]

$$
h_{\mu}^{\nu} \sigma_{\nu ; \alpha}^{\alpha}=\frac{2}{3} h_{\mu}^{\nu} \theta_{; \nu} \quad, h_{\mu}^{\nu} E_{\nu ; \alpha}^{\alpha}=\frac{1}{3} h_{\mu}^{\nu} \rho_{; \nu}
$$

where $h_{\mu \nu}$ is the decomposable tensor defined by the expression $h_{\mu \nu}=g_{\mu \nu}-\frac{1}{u_{\lambda} u^{\lambda}} u_{\mu} u_{\nu}$, and $E_{\nu}^{\mu}=E e_{\nu}^{\mu}, \sigma_{\nu}^{\mu}=$ $\sigma e_{\nu}^{\mu}$. Obviously when the equations (6) are satisfied identically then equations (11)-(4) describe a system of ordinary differential equations, while in general they form a system of partial differential equations. The spacelike constraint equations are essential for the integrability of the silent models and in order the solution to reduce to the Szekeres models [2, 9]. In the following we work with the system (11)-(4) and the constants of integration will be functions independent on the time derivative which are constrained by the system (6).

The integrability of the Szekeres system (1)-(4) has been proved recently with the use of the Darboux polynomial and the Jacobi multiplier methods [10]. Here we study the Szekeres system with two different methods in the search for integrability and the existence of analytical solutions. Specifically we use the symmetry method, group invariant transformations and the singularity analysis. The purpose of this analysis is to study the relationship between the two methods and see how the various solutions, different universes, are related with the symmetries or the movable singularities of the field equations ${ }^{2}$. Both methods have played an important role in gravitational studies while recently they have been applied in modified theories of gravity for the determinant of integrable field equations, for instance see [12 19] and references therein.

The jet-space which is defined by the Szekeres system is $J_{S}=\{\tau, \rho, \theta, \sigma, \mathcal{E}\}$ where $\tau$ is the independent variable and it is a local coordinate of the spacetime. Consider the generator $X$ of a one-parameter point transformation in space $J_{S}$. then

$$
X=\xi\left(\tau, Y^{B}\right) \partial_{t}+\eta^{\rho}\left(\tau, Y^{B}\right) \partial_{\rho}+\eta^{\theta}\left(\tau, Y^{B}\right) \partial_{\theta}+\eta^{\sigma}\left(\tau, Y^{B}\right) \partial_{\sigma}+\eta^{\mathcal{E}}\left(\tau, Y^{B}\right) \partial_{\mathcal{E}}
$$

where $Y^{B}=(\rho, \theta, \sigma, \mathcal{E})$.

However, the Szekeres system can be written as a system of two second-order ordinary differential equations. Without loss of generality we select the two dependent variables to be the energy density, $\rho$, and the electric component, E.

The system of second-order differential equations is

$$
\begin{aligned}
& \frac{d^{2} \rho}{d \tau^{2}}=\Phi(\rho, \mathcal{E}) \quad \text { and } \\
& \frac{d^{2} \mathcal{E}}{d \tau^{2}}=\Psi(\rho, \mathcal{E})
\end{aligned}
$$

where now the expansion rate and the shear are given from the relations

$$
\begin{aligned}
\theta & =-\frac{\dot{\rho}}{\rho} \text { and } \\
\sigma & =\frac{2(\dot{\rho} \mathcal{E}-\rho \dot{\mathcal{E}})}{\rho(\rho+6 \mathcal{E})} .
\end{aligned}
$$

With the use of the latter expressions we observe that any point symmetry, (17), of the Szekeres system is nothing else than a generalised symmetry for the system, (9).

We now consider that the symmetry vector is the generator of a point transformation in $\bar{J}_{S}=\{\tau, \rho, \mathcal{E}\}$. Then

$$
\bar{X}=\xi\left(\tau, Y^{B}\right) \partial_{t}+\eta^{\rho}\left(\tau, Y^{B}\right) \partial_{\rho}+\eta^{\mathcal{E}}\left(\tau, Y^{B}\right) \partial_{\mathcal{E}}
$$

Note that $\bar{J}_{S} \subset J_{S}$, which means that by assuming a point transformation at $\bar{J}_{S}$ we find only a partial number of symmetries for the original system, but, as well see below, that it is sufficient in order to prove the integrability of the system (9) as also to determine an analytical solution.

\footnotetext{
${ }^{2}$ A discussion of these two different methods for the study of integrability can be find in [11].
} 
We find that the admitted Lie point symmetries of the second-order system (9) are

$$
\begin{gathered}
X_{1}=\partial_{\tau}, \\
X_{2}=\frac{\rho \mathcal{E}}{\rho+6 \mathcal{E}}\left(6 \partial_{\rho}-\partial_{\mathcal{E}}\right)
\end{gathered}
$$

and

$$
X_{3}=\tau \partial_{\tau}-\frac{2 \rho(\rho+12 \mathcal{E}) \partial_{\rho}+12 \mathcal{E}^{2} \partial_{\mathcal{E}}}{\rho+6 \mathcal{E}}
$$

which constitute the $A_{1} \otimes_{s} 2 A_{1}$ Lie algebra. $X_{1}$ is the autonomous symmetry and $X_{3}$ is a rescaling symmetry which we see below that the existence of both is important for the singularity analysis.

We apply the coordinate transformation ${ }^{3}$ to (9),

$$
\rho=\frac{6}{(1-x) y^{3}}, \mathcal{E}=\frac{x}{y^{3}(x-1)},
$$

and the Szekeres system takes the simpler form,

$$
\ddot{x}+2 \frac{\dot{y}}{y} \dot{x}-\frac{3}{y^{3}} x=0
$$

and

$$
\ddot{y}+\frac{1}{y^{2}}=0
$$

From (15) we find the conservation law

$$
I_{1}=\dot{y}^{2}-2 y^{-1}
$$

which gives

$$
\int \frac{d y}{\sqrt{I_{1}+2 y^{-1}}}=\tau-\tau_{0}
$$

while, when $I_{1}=0$, we have the closed-form solution

$$
y(\tau)=\left(\frac{3 \sqrt{2}}{2}\left(\tau-\tau_{0}\right)\right)^{\frac{2}{3}} .
$$

Therefore, when we substitute $y(\tau)$ into (14), we obtain a second-order linear equation which is maximally symmetric and integrable. In the limit in which $I_{1}=0$, we find the closed-form solution

$$
x(t)=x_{1}\left(\tau-\tau_{0}\right)^{\frac{2}{3}}+x_{2}\left(\tau-\tau_{0}\right)^{-1} .
$$

Moreover we observe that the Szekeres system, (14), (15), follows from the variation of the Action integral

$$
S=\int L(x, \dot{x}, y, \dot{y}) d \tau
$$

where $L(x, \dot{x}, y, \dot{y})$ is point-like Lagrange function

$$
L(x, \dot{x}, y, \dot{y})=y \dot{x} \dot{y}+x \dot{y}^{2}-x y^{-1} .
$$

\footnotetext{
3 The new variables that we have considered are related with the q-scalar variables which have been introduced in [7]. Specifically it holds $y^{3}=6 \rho_{q}^{-1}, x=\Delta^{(\rho)}\left(1+\Delta^{(\rho)}\right)^{-1}$, where by definition it follows that $\theta_{q}=3 \dot{y} y^{-1}$ and $\dot{x}(1-x)^{-1}=-3 \sigma$.
} 
The Lagrangian is autonomous and is invariant under the action of the symmetry vector $X_{1}$. Hence Noether's second theorem tells us that the corresponding conservation law is the "energy",

$$
H \equiv \frac{p_{x} p_{y}}{y}-\frac{x}{y^{2}}\left(p_{x}\right)^{2}+\frac{x}{y}=h
$$

where $p_{x}$ and $p_{y}$ are the normal momenta, that is,

$$
y^{2} \dot{x}=y p_{y}-2 x p_{x} \text { and } y \dot{y}=p_{x} .
$$

This is a Lagrange description of the field equation (1)-(4) for a specific lapse, derivative. However as it is well known the field equations are invariant under the definition of another lapse i.e. $d \tau \rightarrow N\left(\tau^{\prime}\right) d \tau^{\prime}$, where $N\left(\tau^{\prime}\right)$ is an arbitrary function. However that it is not true for the Action Integral (18) and the corresponding Euler-Lagrange equations will describe the Szekeres system. The description that we have presented here will be used in order to consider the field equations as equations of motion of a particle where methods of Classical Mechanics can be studied. A discussion on the conformal equivalent Lagrangians can be found in [21].

In terms of Noether's Theorem we can say that the conservation law, $I_{1}$, is generated by a generalized symmetry and specifically a contact symmetry. It holds that $\dot{I}_{1}=\left\{I_{1}, H\right\}=0$, which means that the dynamical system, (14), (15), is Liouville integrable. For completeness we find that the system, (14), (15) admits another linear-in-the-momentum conservation law which is nonautonomous and is generated by the Lie symmetry vector $X_{3}$.

From (20) with the use of (16) we find that the solution of the Hamilton-Jacobi Equation provides us with the Action

$$
S(x, y)=-\frac{\left(I_{1} x+h\right)}{I_{1}} \sqrt{\left(I_{1} y^{2}+2 y\right)}-\frac{h}{\left(I_{1}\right)^{\frac{3}{2}}} \ln \left(\frac{I_{1} y+1}{\sqrt{I_{1}}}+\sqrt{\left(I_{1} y^{2}+2 y\right)}\right) \quad \text { for } \quad I_{1} \neq 0
$$

and

$$
S(x, y)= \pm \sqrt{2 y} \varepsilon x+h y^{\frac{2}{2}} \text { for } \quad I_{1}=0 .
$$

From (21) the system of first-order ordinary differential equations is

$$
\dot{x}=-\frac{\left(I_{1} y+3\right) x-h y}{y \sqrt{y\left(I_{1} y+2\right)}} \dot{y}=-\frac{\sqrt{y\left(I_{1} y+2\right)}}{y},
$$

that is,

$$
\frac{d y}{d x}=\frac{\sqrt{y\left(I_{1} y+2\right)}}{\left(I_{1} y+3\right) x-h y} .
$$

In the simplest scenario, $h=0$, it follows that $\int \sqrt{\frac{I_{1} y+2}{y}} d y=\ln x$. If we see the system (11)-(4) as ordinary differential equations then the latter solution corresponds to the Class II solution of [22]. However in general the integration constants, and the solution, are constrained by the spacelike constraints (6)

We now continue our analysis with the singularity analysis of the system, (14, 15). We apply the ARS algorithm which is described in [23]. We omit the details of the calculations. We find the leading-order behaviours:

$$
x_{D}(z)=x_{0} z^{-1}, y_{D}(z)=y_{0} z^{\frac{2}{3}}
$$

and

$$
\bar{x}_{D}(z)=x_{0} z^{\frac{2}{3}}, \bar{y}_{D}(z)=y_{0} z^{\frac{2}{3}}
$$

where $z=\tau-\tau_{0}, y_{0}$ satisfies the algebraic equation $y_{0}^{3}=\frac{9}{2}$ and $x_{0}$ is a constant of integration. All terms are dominant. From (17) we can see that the leading-order powers solve the field equations.

The resonances are found to be

$$
s_{0}=-1, s_{1}=0, s_{2}=\frac{2}{3}, s_{3}=\frac{5}{3},
$$

while for the leading-order behaviours in (26) the resonances are

$$
\bar{s}_{0}=-1, \bar{s}_{1}=0, \bar{s}_{2}=\frac{2}{3}, \bar{s}_{3}=-\frac{5}{3} .
$$


Note that the resonances are the same for every $y_{0}^{3}=\frac{9}{2}$. Last but not least we performed the consistency test and we found that the field equations pass the singularity test. That means that the solution can be described in terms of a Laurent expansion.

The singularity analysis provides a different kind of integrability from that of the symmetries. For instance, while the harmonic oscillator is a well-known integrable system from the symmetry point of view, it fails to pass the singularity test. However, as discussed in [11], the singularity analysis is coordinate dependent. It is important to realize that the dependence of the results of the singularity analysis upon a coordinate representation does not mean that the integrability in terms of singularity is coordinate dependent. This means that for other coordinates maybe the solution can be written in terms of a Laurent expansion.

From the resonances, (27), we have that the Laurent expansion is given by a Right Painlevé Series, while a LeftRight Painlevé Series describes the solution with resonances (28). However, the two dominant terms and the two different sets of resonances do not mean that there are different expansions which describe the solution. Actually it is only one solution. That can be seen easily from the position of the constants of integration for both the expansions which are the same. Moreover it is important to mention that the dominant term $x_{D}$ is the special solution in which $h=0$.

However, the special solutions, (25) and (26), are unstable, that is, under a perturbation around the solutions or if the initial conditions change such the values of $I_{1}$ and $h$ change, they fail to describe the system. Of course that can be seen from the singularity analysis because in the Right Painlevé Series the new terms which are given from the expansion are becoming more dominant as we go further from the movable singularity. However, in the special case in which the initial conditions change such that $I_{1}$ continues to be zero, the special solution $\bar{x}_{D}=x_{0} \tau^{2 / 3}$ continues to describe the system, which means that it is stable. That property for this special solution is related with the existence of the Left part for the Left-Right Painlevé Series.

Last but not least in order to relate the singularity analysis with the symmetry vectors of the original system we want to say that the special solution (25) is an invariant solution in the sense that it follows from the zeroth-order invariants of the Lie symmetry vector $X_{3}$. These two methods both provide the conservation law for the Szekeres system and show that there is a direct equivalence of these two methods with the analysis using the method of the Darboux polynomial and that of the Jacobi multiplier. However, what it is more interesting is the existence of a point-like Lagrangian which describes the evolution of the gravitational field equations, while the conservation laws are Noetherian.

The two conservation laws with the use of the original coordinates $(\rho, \theta, \sigma, \mathcal{E})$ can be presented as the following algebraic system

$$
I_{1}=\frac{2^{\frac{2}{3}}\left[(3 \sigma+\theta)^{2}-3(\rho+6 \mathcal{E})\right]}{3^{\frac{4}{3}}(\rho+6 \mathcal{E})^{\frac{2}{3}}}
$$

and

$$
h=\left(\frac{4}{3}\right)^{\frac{1}{3}} \frac{18 \mathcal{E}^{2}+3 \rho \sigma(3 \sigma+\theta)+\mathcal{E}(3 \rho-2 \theta+6 \sigma(6 \sigma+\theta))}{\rho(\rho+6 \mathcal{E})^{\frac{2}{3}}}
$$

which defines the surfaces where the system evolves.

Furthermore, the Ricci scalar $R$ and the Weyl curvature invariant $\Psi_{2}$ are related with the variables $\{x, y\}$ as $x=$ $6 \frac{\Psi_{2}}{R}, y^{-3}=\Psi_{2}-\frac{1}{6} R$ where the homogeneous limit provides $(x, y) \rightarrow(0,0)$ [7]. We can see that the (special) power-law solutions (25), (26) do not provide a homogeneous universe.

In a forthcoming work we intend to study the quantization of the Szekeres system by using the point-like Lagrangian that we found in the spirit of the minisuperspace approach of quantum cosmology and also to extend our analysis in the case of the silent universe.

\section{Acknowledgments}

We acknowledgement the anonymous referees for their comments and suggestions which improved the quality of this work. AP acknowledges financial support of FONDECYT grant no. 3160121. AP thanks the Durban University of Technology for the hospitality provided while this work was performed. PGLL acknowledges the National Research Foundation of South Africa and the University of KwaZulu-Natal for financial support. The views expressed in this 
letter should not be attributed to either institution.

[1] M. Bruni, S. Matarrese and P. Ornella, Astro. J. 445, 958 (1995)

[2] H. van Elst, C. Uggla, W.M. Lesame, G.F.R. Ellis and R. Maartens, Class. Quantum Grav. 14, 1151 (1997)

[3] H. Mutoh, T. Hirai and K-i Maeda, Phys. Rev. D 55, 3276 (1997)

[4] N. Mustapha, G.F.R. Ellis, H. van Elst and M. Marklund, Class. Quantum Grav. 17, 3135 (2000)

[5] P. Szekeres, Commun. Math. Phys. 41, 55 (1975)

[6] A. Barnes and R.R. Rowlingson, Class. Quantum Gravi. 6, 949 (1989)

[7] R. A. Sussman and K. Bolejko, Class. Quantum Grav. 29, 065018 (2012)

[8] P.S. Apostolopoulos, Szekeres models: a covariant approach, [preprint: arXiv:1611.04569.

[9] R. Maartens, W. Lesame and G.F.R. Ellis, Phys.Rev. D 55, 5219 (1997)

[10] A. Gierzkiewicz and Z.A. Golda, J. Nonl. Math. Phys. 24, 494 (2016)

[11] A. Paliathanasis and P.G.L. Leach, Int. J. Geom. Meth. Mod. Phys. 13, 1630009 (2016)

[12] S. Capozziello, R. de Ritis, C. Rubano and P. Scudellaro, Riv.Nuovo Cim. 19, N4 1 (1996)

[13] S. Capozziello, N. Frusciante and D. Vernieri, Gen. Rel. Gravit. 44, 1881 (2012)

[14] N. Dimakis, T. Christodoulakis and P.A. Terzis, J. Geom. Phys. 77, 97 (2014)

[15] B. Vakili, Ann. Phys. 19, 359 (2010)

[16] A. Paliathanasis, Class. Quantum Gravit. 33, 075012 (2016)

[17] J. Demaret and C. Scheen, J. Math. Phys. A: Math. Gen. 2959 (1996)

[18] S. Cotsakis, J. Demaret, Y. De Rop and L. Querella, Phys. Rev. D 48, 4595 (1993)

[19] A. Paliathanasis and P.G.L. Leach, Phys. Lett. A 380, 2815 (2016)

[20] A. Paliathanasis, J.D. Barrow and P.G.L. Leach, Phys. Rev. D 94, 023525 (2016)

[21] M. Tsamparlis, A. Paliathanasis, S. Basilakos and S. Capozziello, Gen. Relativ. Gravit. 45, 2003 (2013)

[22] S.W. Goode and J. Wainwright, Phys. Rev. D 26, 3315 (1982)

[23] A. Ramani, B. Grammaticos and T. Bountis, Phys. Rep. 180, 159 (1989) 